\title{
LA PROSTAGLANDINE E1: DONNEES PHYSIOLOGIQUES ET PHARMACOLOGIQUES
}

\author{
Michel ETCHEVERRY
}

\author{
Clinique Bordeaux-Nord - Service Uro-Andrologie - 13, Rue Claude Boucher - 33300 BORDEAUX \\ G.E.T.S.M. (Groupe d'Etude des Troubles Sexuels Masculins), Université Bordeaux II
}

\section{PROSTAGLANDIN E1 : PHYSIOLO- GICAL AND PHARMACOLOGI-} CAL DATA. Prostaglandins (PGs) discovered in 1930 are derived from prostanoic acid and are present in the prostate and seminal vesicles. Every tissue is capable of producing PGs from cell membranes by the action of cyclooxygenase on arachidonic acid. The half-life of PGs is very short, being less than five minutes. Prostaglandin E1 or PGE1 (Alprostadil : Prostine VR, Upjohn : $\mathrm{C}_{20} \mathrm{H}_{34} \mathrm{O}_{5}$ ) exerts a powerful vasodilatory effect upon peripheral arteries as well as stimulating intestinal and uterine smooth muscle. A strong vasodilatory effect has also been demonstrated in vitro on cavernosal arteries and erectile tissue, via antagonism of adrenergic receptors, by Hedlund and Anderson. PGEl which is biosynthesised in the corpus cavernosum, is metabolised by the liver, Kidneys and lungs, and destroyed by prostaglandin dehydrogenase. The first therapeutic trial of PGE1 for organic impotence was reported by Ishii in 1986, since which time many groups, especially in Europe, have reported large clinical series. This review considers the experience of some of these workers. Key-words : Postaglandin El - Diagnosis Treatment - Male impotence. Andrologie 19911 : 126-127.

Les Prostaglandines tiennent leur nom de la découverte, par Von Heuler en 1930, de dérivés de l'acide prostanoïque dans le liquide séminal et la prostate. Elles sont présentes dans de nombreux tissus, et du fait de leur caractère ubiquitaire, on les a considèrées comme des modulateurs de toutes les activités cellulaires. Les voies métaboliques de synthèse sont multiples et complexes, et le liquide séminal est le seul site où elles sont stockées. Ailleurs, elles sont immédiatement dégradées après avoir agi.

Ce sont des lipides simples, dérivés de trois acides gras insaturés, à 20 atomes de carbone. La nomenclature biochimique préfère le terme d'eicosanoïdes. Le principal précurseur est l'acide arachidonique (acide eicosatetranoïde). Les Prostaglandines de série 1 dérivent de l'acide eicosatrienoïque. Leur structure a été définie par le Suèdois Bergström. L'hormone qui nous intéresse dérive de l'action de la cyclo-oxygénase sur l'acide gras précurseur, lui-même détaché des membranes cellulaires par une phospholipase. Toutes les cellules produisent des eicosanoìdes dont la nature dépend de leur équipement enzymatique. Leur durée de vie est très courte; elles sont fabriquées à la demande pour agir immédiatement, au lieu même de leur production. Ce sont des médiateurs locaux dont l'existence est inférieure à 5 minutes. Les Prostaglandines contrôlent :

- la contraction des muscles lisses,

- les sécrétions glandulaires,

- la résorption de l'eau et des électrolytes,

- la neurotransmission,

- la régulation des plaquettes,

- l'induction de la fièvre,

- celle des réponses inflammatoires.

Ces effets peuvent être opposés suivant les variétés et s'inverser selon les doses. Le premier effet fut mis en évidence sur le muscle utérin, et l'on a démontré leur rôle dans les processus de reproduction chez le mâle et la femelle, dans le déclenchement de l'accouchement et de l'avortement. Enfin, les Prostaglandines E1 et E2 jouent un rôle dans le contrôle de la douleur et augmentent la sensibilité des nocicepteurs. La Prostaglandine El (PGE1) ou Alprostadil, est commercialisée sous le nom de Prostine VR par le laboratoire Upjohn. C'est un eicosanoïde de formule $\mathrm{C} 20 \mathrm{H} 34 \mathrm{O}$ : Acide (II alpha, 13E, 15S) 11-15 Dihydroxy-9-Oxoprost-13-EN-1-Ö̈que. La PG E1 est surtout utilisée pour assurer le maintien temporaire de la perméabilité du canal artériel jusqu'à l'intervention chez les nouveauxnés porteurs d'une cardiopathie congénitale ducto-dépendante.

\section{PROPRIETES PHARMACODYNAMIQUES}

L'Alprostadil n'a pas la même action dans les diverses espèces animales. Ses effets peuvent être différents en fonction des doses employées, des organes étudiés ou de la voie d'administration locale ou générale.

\section{Action sur le système cardio-vasculaire}

En perfusion veineuse, la PGE1 provoque céphalées, rougeurs du visage, bouffées de chaleur, du fait d'un pouvoir vaso-dilatateur puissant sur les artérioles périphériques.

\section{Action sur la musculature lisse}

Au niveau bronchique, elle est bronchodilatatrice, alors qu'elle stimule la motricité intestinale et les contractions utérines. Elle est utilisée dans le protocole d'interruption de la grossesse par la Mife pristone ( $\mathrm{Ru} 486$ ); mais elle ne doit pas être confondue avec les analogues de la PGE2, entrant également dans la séquence d'I.V.G. et pour lesquels il existe des contreindications bien établies, du fait des complications observées.

Dans le corps caverneux, Hedlund et Anderson (2) ont démontré un effet vaso-dilatateur sur les artères caverneuses et le tissu érectile, qu'ils attribuent à un effet antagoniste sur les récepteurs adrénergiques, bloquant la libération de norepinéphrine au niveau des terminaisons nerveuses.

\section{Action sur le système nerveux central}

Elle est à l'étude, mais on a noté une élèvation de la température chez les enfants mis sous perfusion veineuse. La PGE1 est présente dans tous les tissus à innervation sympathique et participe au contrôle de la sécrétion des amines.

\section{PROPRIETES PHARMACOCINETIQUES}

L'Alprostadil, après injection intracaverneuse, diffuse très rapidement dans tous les organes, sauf dans le système nerveux central, où elle ne pénètre guère. Sa demi-vie est inférieure à 5 minutes, car elle est métabolisée très vite par le rein, le foie, et surtout le poumon qui en capte l'essentiel au premier passage (4). Sa destruction est assurée par la $15 \mathrm{OH}$ Prostaglandine déshydrogénase, et les métabolites inactifs sont éliminés par le rein dans les 24 heures.

\section{EFFETS INDESIRABLES}

Surtout utilisée en perfusion veineuse ou même intra-aortique chez les nouveaux-nés porteurs de malformations cardiaques, la PGE1 entraîne dans cette indication : fièvre, flush, troubles du rythme cardiaque, cedèmes, hypotension. 


\section{BIOSYNTHESE}

Les Prostaglandines sont présentes dans tous les tissus. Le corps caverneux en produit plusieurs, même in vitro (5). Chez l'homme, Hamberg a démontré la présence de fortes concentrations dans les vésicules séminales et le liquide séminal (1).

\section{PROSTAGLANDINE E1 ET IMPUISSANCE}

A la suite des travaux précités de Hedlund (2) Ishii a réalisé le premier essai d'utilisation intracaverneuse en clinique humaine et a rapporté les premiers résultats, à Prague, lors du deuxième Congrès de l'International Society for Impotence Research. (3) Depuis, en particulier en Europe, de nombreuses équipes ont acquis une expérience importante dans ce domaine. Plusieurs d'entre elles la rapportent dans les articles suivants.

\section{REFERENCES}

1- Hamberg M : Biosynthesis of Prostaglandin E1 by human seminal vesicles. Lipids - 1976; 11 : 240-245.

2 - Hedlund H., Anderson K.E : Contraction and relaxation induced by some prostanoïds in isolated human penile erectile tissue, and cavernous artery. J. Urol. 1985; 134 : 1245-1249.

3 - Ishii N. Watanabe H. Irisawa C., Kiruchi Y. : Therapeutic trial with Prostaglandin El for organic impotence - Prague -The second world meeting on impotence, 1986, June 17-20.

4 - Junemann K.P., Alken P : Pharmacotherapy of erectile dysfunction : a review. - Int. J. impotence Res. 1989, 1,71-93.

5 - Roy A.C., Tans M., Kottegoda S.R. Ratnam S.S : Ability of human corpora cavernosa muscle to generate prostaglandins and thromboxanes in vitro - I.R.C.S. - Med. Sci. 1984, $12: 608-611$.
RESUME. Les PROSTAGLANDINES ont été découvertes en 1930. Elles dérivent de l'acide prostanoïque et sont présentes dans la prostate et les vésicules séminales. Elles sont produites à partir des membranes cellulaires de tous les tissus, par action de la cyclo-oxygénase sur l'acide arachidonique. Leur durée de vie est inférieure à 6 minutes. La PGE1 ou ALPROSTADIL (PROSTINE VR® - Upjohn) a un effet vaso-dilatateur très puissant sur les artères caverneuses et le tissu érectile : c'est un antagoniste des récepteurs adrénergiques. Elle est métabolisée dans le foie, le rein et le poumon, et détruite par la $15 \mathrm{OH}$ PROSTAGLANDINE DESHYDROGENASE. Le premier essai en clinique humaine dans l'impuissance organique a été rapporté par ISHII en 1986. Mots clés : Prostaglandine E1 - Physiologie - Pharmacologie. Andrologie 1991 - 1 : 126-127. 